\title{
Position of the Academy of Nutrition and Dietetics: Malnutrition (Undernutrition) Screening Tools for All Adults
}

\author{
Annalynn Skipper ${ }^{\mathrm{a}} \quad$ Anne Coltman $^{\mathrm{b}}$ Jennifer Tomesko ${ }^{c}$ Pamela Charney $^{\mathrm{d}}$ Judith Porcari ${ }^{\mathrm{e}}$ \\ Tami A. Piemonte ${ }^{f}$ Deepa Handu ${ }^{f}$ Feon W. Cheng ${ }^{f}$ \\ ${ }^{a}$ American Medical Association, Chicago, IL, USA; ${ }^{b}$ Trinity Health, Melrose Park, IL, USA; ${ }^{\circ}$ Rutgers University, School of

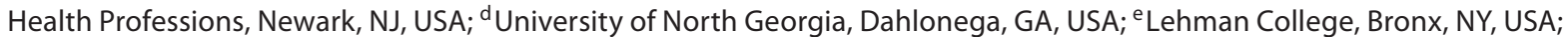 \\ ${ }^{f}$ Academy of Nutrition and Dietetics, Evidence Analysis Center, Chicago, IL, USA
}

\begin{abstract}
It is the position of the Academy of Nutrition and Dietetics that, based upon current evidence, the Malnutrition Screening Tool should be used to screen adults for malnutrition (undernutrition) regardless of their age, medical history, or setting. Malnutrition (undernutrition) screening is a simple process intended to quickly recognize individuals who may have a malnutrition diagnosis. While numerous malnutrition screening tools are in use, their levels of validity, agreement, reliability, and generalizability vary. The Academy of Nutrition and Dietetics reviewed the body of evidence sup-
\end{abstract}

porting malnutrition screening tools and determined a single tool for identifying adults in all settings who may have malnutrition, regardless of their age or medical history. The Nutrition Screening for Adults Workgroup conducted a systematic review of the most robust evidence to promote using the highest-quality malnutrition screening tool available.

(C) 2020 by the Academy of Nutrition and Dietetics. Reprinted from J Acad Nutr Diet. 2020;120(4):709-713 with permission from Academy of Nutrition and Dietetics 2020
Position Statement

It is the position of the Academy of Nutrition and Dietetics that, based upon current evidence, the Malnutrition Screening Tool should be used to screen adults for malnutrition (undernutrition) regardless of their age, medical history, or setting.

Malnutrition (undernutrition) among adults aged 19 years through old age is a common nutrition problem [1]. Because not all individuals with malnutrition have direct access to registered dietitian nutritionists (RDNs), screening to identify those who may have malnutrition or be at risk for malnutrition is a routine part of the intake or admission process in community and health care settings. Nutrition screening, as described in the Nutrition Care Process, is separate and distinct from nutrition assessment $[2,3]$, and is performed by nurses; medical assistants; and nutrition and dietetics technicians, registered. Based on a predetermined score, patients or clients are referred to an RDN for nutrition assessment, an in-depth process that is a licensed function in many states. Nutrition assessment may involve verifying some of the information obtained during screening and then obtaining additional information necessary for a malnutrition diagnosis [3]. Widespread use of valid and reliable malnutrition screening tools increases the likelihood that individuals referred to an RDN for assessment will have a malnutrition diagnosis. Conversely, using valid and reliable tools avoids unnecessary referrals of people who do not have malnutrition. 


\section{Position Focus}

Malnutrition occurs in health care settings, and in communities where people suffer from food insecurity and hunger. Thus, this position applies in all settings where food assistance and nutrition services are available. This position is based on a comprehensive systematic review $[4,5]$ and is intended to provide RDNs and all other health professionals with validity, agreement, reliability, and generalizability data for six malnutrition screening tools supported by the largest number of studies (Malnutrition Screening Tool [MST] [6], Malnutrition Universal Screening Tool [7], Mini Nutritional Assessment-Short Form [8, 9], Short Nutritional Assessment Questionnaire [10], Mini Nutritional Assessment-Short Form Body Mass Index [11], and Nutrition Risk Screening 2002) [12]. This position supports using a single tool to identify adults who may be malnourished regardless of age, acute and chronic medical conditions, or settings where care is received.

\section{Position Statement Development Process}

The Academy's Nutrition Screening for Adults Workgroup systematically reviewed validation studies for malnutrition screening tools published in the peer-reviewed literature from January 1997 through July 2017 and used the results as a basis for this po- sition $[4,5]$. The Workgroup included tools that met the Academy's 2011 definition of nutrition screening (Table 1), which was created for an earlier version of this project [13]. As in the earlier systematic review, the Workgroup sought quick and easy screening tools, defined as requiring fewer than 10 minutes to complete. For the current position, the requirement for robust data necessitated inclusion of tools with adequate supporting evidence, defined as greater than four validation studies. The Workgroup considered the overall validity, agreement, and reliability results and grades of supporting evidence for each tool, then generalizability of each tool to the widest variety of medical diagnoses or age groups and settings and ranked the tools accordingly (Table 2). The position paper was posted for public comment before publication.

\section{Position}

It is the position of the Academy of Nutrition and Dietetics that, based upon current evidence, the MST should be used to screen adults for malnutrition (undernutrition) regardless of their age, medical history, or setting.

Ferguson and colleagues [6] developed the MST to detect malnutrition or risk for malnutrition. Between 1999 and 2017, the MST was validated in acute, long-term, rehabilitation, and ambulatory

Table 1. The Academy of Nutrition and Dietetics' definition and key considerations for nutrition screening

Definition: Nutrition screening is the process of identifying patients, clients, or groups who may have a nutrition diagnosis and benefit from nutrition assessment and intervention by a registered dietitian nutritionist (RDN).

Key considerations:

- May be conducted in any practice setting as appropriate

- Tools should be quick, easy to use, valid, and reliable for the patient population or setting

- Tools and parameters are established by RDNs, but the screening process may be carried out by nutrition and dietetics technician, registered and other trained personnel

- Nutrition screening and rescreening should occur within an appropriate time frame for the setting.

(Reprinted with permission from Skipper and colleagues [13]; ๔ 2012 American Society for Parenteral and Enteral Nutrition.)

Table 2. Validity, agreement, reliability, generalizability, and strength of evidence of adult malnutrition (undernutrition) screening tools

\begin{tabular}{|c|c|c|c|c|c|c|c|c|c|}
\hline \multicolumn{6}{|l|}{ Validity ${ }^{a}$} & \multirow[t]{2}{*}{ Agreement $^{a}$} & \multirow[t]{2}{*}{ Reliability ${ }^{a}$} & \multirow{2}{*}{$\begin{array}{l}\text { Generaliz- } \\
\text { abilityc }^{c}\end{array}$} & \multirow{2}{*}{$\begin{array}{l}\text { Evidence Grade, } \\
\text { Strength }^{d}\end{array}$} \\
\hline Tool & Sensitivity & Specificity & $\begin{array}{l}\text { Positive } \\
\text { Predictive } \\
\text { Value }\end{array}$ & $\begin{array}{l}\text { Negative } \\
\text { Predictive } \\
\text { Value }\end{array}$ & $\begin{array}{l}\text { Overall } \\
\text { Validity }^{b}\end{array}$ & & & & \\
\hline MSTe & Moderate & Moderate & Moderate & Moderate & Moderate & Moderate & Moderate & Good & I, Good/strong \\
\hline MUST $^{f}$ & Moderate & Moderate & Moderate & High & High & Moderate & Moderate & Fair & II, Fair \\
\hline MNA-SFg & Moderate & Moderate & Low & Moderate & Moderate & Low & Moderate & Fair & II, Fair \\
\hline SNAQ ${ }^{h}$ & Moderate & High & Low & High & Moderate & - & Moderate & Fair & II, Fair \\
\hline MNA-SF-BMI' & Moderate & Moderate & Moderate & High & High & Moderate & - & Limited & II, Fair \\
\hline NRS-2002 & Moderate & High & Moderate & Moderate & Moderate & Moderate & - & Limited & II, Fair \\
\hline
\end{tabular}

a Sensitivity, specificity, positive predictive value, negative predictive value cutoffs: High: $90 \%$ to $100 \%$, moderate: $80 \%$ to $\leq 89 \%$, low: $\leq 79 \%$; agreement and reliability K. Cutoffs: High: 0.8 to 1; moderate: 0.6 to $\leq 7.9$; low: $\leq 5.9$. b See Figure 3 in Skipper and colleagues [4] for the algorithm to determine the overall validity. ${ }^{C}$ The Workgroup determined generalizability based on usefulness of each tool across the broadest array of adult age groups, locations, diseases, and treatments according to evidence. ${ }^{d}$ Elements considered in the evidence grade include quality of the evidence, consistency of results across studies, quantity of studies, and number of subjects, clinical impact of outcomes, and generalizability to population of interest [14]. ${ }^{e}$ MST = Malnutrition Screening Tool. ${ }^{\mathrm{f}}$ MUST = Malnutrition Universal Screening Tool. ${ }^{\mathrm{g}}$ MNA-SF = Mini Nutritional Assessment-Short Form. ${ }^{\mathrm{h}} \mathrm{SNAQ}=\mathrm{Short}$ Nutritional Assessment Questionnaire. ' MNA-SF-BMI = Mini Nutritional Assessment-Short Form Body Mass Index. ${ }^{j}$ NRS-2002 = Nutrition Risk Screening 2002. 
care and oncology clinics in at least nine different countries [1533]. These studies revealed that the MST exhibited a moderate degree of validity, a moderate degree of agreement, and a moderate degree of inter-rater reliability in identifying malnutrition risk in adults (Table 2). The strength of evidence for the MST is Grade I, good/strong with good generalizability $[4,5,14]$. Some of the other tools also had high or moderate validity, agreement, or reliability, but were not supported by Grade I evidence or good generalizability $[4,5]$.

\section{Implication for Practitioners}

While disease-, age-, or setting-specific malnutrition screening tools exist, most organizations where malnutrition screening occurs have clients or patients of different ages with one or more medical problems and provide nutrition care in a variety of settings. The value of using different screening tools for individuals with different personal characteristics is unclear, and subject to practical limitations. It is appropriate to implement the screening tool that will most accurately identify adults who may have malnutrition. Institutions and programs that implement the MST should have data available to consistently compare the populations screened for malnutrition, predict the resources for needed treatment, and support research.

Based upon the best available evidence $[4,5]$, the Academy advocates that RDNs:

- Assume a strong leadership role in implementing the MST. A benefit to society occurs if individuals who may have malnutrition obtain nutrition assessment and intervention services from an RDN. This benefit is not without cost because of the time required for an $\mathrm{RDN}$ to complete a nutrition assessment. Thus, the impact of changes to screening procedures affects the amount of RDN time available to provide other required nutrition services and provides justification for RDNs to select and oversee implementation of malnutrition screening tools.

- Implement the MST without changes to the wording of the questions or the scoring system for referrals as originally presented [6]. Adding items, modifying questions, or interpreting scores differently than intended by the authors of the tool should be avoided, as these changes invalidate the MST. Individual patients or clients with an MST score of $\geq 2$ should achieve the greatest benefit from an RDN referral.

- Provide ongoing training to paraprofessionals who administer the MST and monitor the impact of the screening and referral process by summarizing data from individuals with malnutrition.

- Abandon all unvalidated malnutrition screening tools (eg, pressure injury and illness severity tools), including tools that were validated, then modified without rigorous re-validation against a standard definition of malnutrition.
- Discourage strongly the development of new screening tools in favor of further validating existing tools [13], especially in adults between the ages of 19 and 49 years, over age 90 years, and in community and long-term care settings.

- Research the costs and outcomes of the malnutrition screening procedure. Minimal data exist on the financial implications of the proper identification of patients who do or do not have malnutrition or the costs of the screening procedure [4, 5]. Obtaining these data could enable the projection of malnutrition assessment and intervention cost and should be a research priority.

- Research the minimum level of education and training needed to accurately administer the MST and develop education and training materials to facilitate consistency among users.

- Develop partnerships with patient advocacy groups, other health care professional organizations, and policy makers to implement the MST.

\section{Conclusions}

The amount and quality of evidence for validated malnutrition screening tools has grown considerably since an earlier review of the topic. This additional evidence supports using a single tool, the MST, to screen all adults for malnutrition (undernutrition) in all settings where malnutrition screening occurs. Replacing other malnutrition screening tools, especially those not rigorously validated, with the MST is expected to identify persons with malnutrition and provide consistent data to support nutrition practice and policy.

\section{Statement of Potential Conflict of Interest}

The authors of the paper have no conflicts to disclose. A. Skipper is an employee of the American Medical Association (AMA), but the ideas expressed in this position are those of the Academy of Nutrition and Dietetics and do not reflect AMA policy.

\section{Funding/Support}

There is no funding to disclose.

\section{Acknowledgements}

The authors thank the reviewers for their many constructive comments and suggestions. The reviewers were not asked to endorse this position or the supporting paper.

\section{References}

The references are available at www.karger.com/doi/10.1159/000516528. 